\title{
Computer Assisted Quantitative Analysis of Deformities of the Human Spine
}

\author{
B. Verdonck ${ }^{1}$, R. Nijlunsing ${ }^{1}$, F. A. Gerritsen ${ }^{1}$, J. Cheung ${ }^{2}$, D. J. Wever ${ }^{2}$, \\ A. Veldhuizen ${ }^{2}$, S. Devillers ${ }^{3}$, S. Makram-Ebeid ${ }^{3}$ \\ ${ }^{1}$ Philips Medical Systems Nederland B.V., P.O.Box 10.000, NL-5680 DA Best, \\ The Netherlands, Bert.Verdonck@best.ms.philips.com \\ http://www.medical.philips.com \\ ${ }^{2}$ Academic Hospital Groningen, P.O. Box 30.001, \\ NL-9700 RB Groningen, The Netherlands \\ ${ }^{3}$ Laboratoires d'Electronique Philips S.A.S., P.O. Box 15, \\ F-94453 Limeil-Brevannes cedex, France
}

\begin{abstract}
Nowadays, conventional X-ray radiographs are still the images of choice for evaluating spinal deformaties such as scoliosis. However, digital translation reconstruction gives easy access to high quality, digital overview images of the entire spine. This work aims at improving the description of the scoliotic deformity by developing semi-automated tools to assist the extraction of anatomical landmarks (on vertebral bodies and pedicles) and the calculation of deformity quantifying parameters. These tools are currently validated in a clinical setting.
\end{abstract}

\section{Introduction}

The interest in three dimensional (3D) analyses of spinal deformities is increasing over the last decades. This evolution is partially due to the progress of digital imaging technology and automated image processing. The description and quantification of the geometry of scoliosis, a complex 3D deformity of the spine, is essential and will assist the clinician in the accurate and reliable follow up of natural history, brace and operative treatment.

Computer Tomography (CT) imaging could give immediate access to 3D information. However, it does not allow the patient to be in a natural standing posture and it would expose him to higher X-ray dose. Optical and opto-electronic image capture is without any risk for the patient but yields limited accuracy and gives only indirect information about the deformity of the vertebral column. Large focus, long film X-ray radiographs are still the images of choice for evaluating deformities of the entire spine in general and scoliosis more specifically.

We have developed a digital method for creating complete overview images of the human vertebral column [1]. The image is reconstructed from a series of overlapping $\mathrm{X}$-ray images acquired with a dedicated protocol on a conventional image intensifier based digital X-ray system (with constant translation speed and frame acquisition rate). Successive images are matched and merged into one overview image. These overviews have demonstrated improved image quality for equivalent $\mathrm{X}$-ray dose as compared to 
conventional film-based techniques (figure 1). This reconstruction technique is commercially available as one of the software modules of the EasyVision product line of Philips Medical Systems.
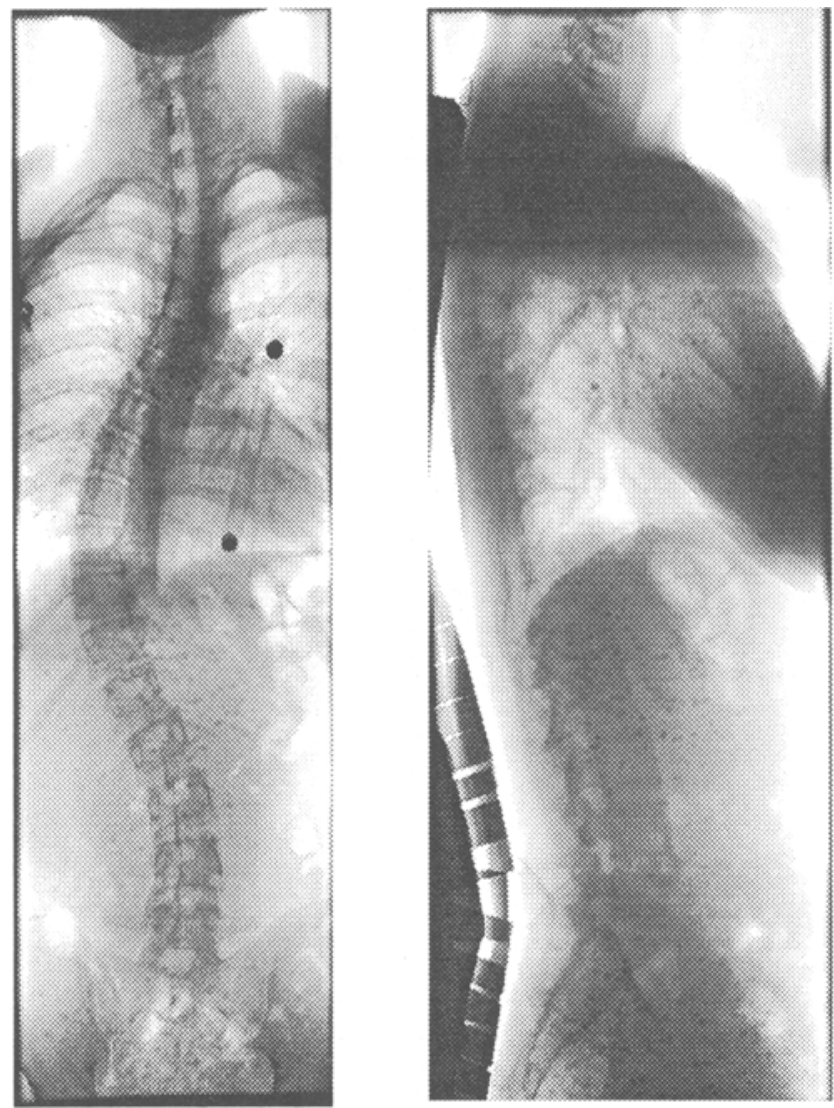

Fig. 1. Digital overview image of the spine, constructed from a translated series of digital images with optimized exposure and contrast. Fontal and lateral acquisitions.

The EasyVision Spine software includes utilities for the measurement of Cobb's angle, vertical alignment and femur height difference. At the end of a previous paper [1] some attention was drawn to the accuracy of measurements on translation reconstructed images and to the limited precision of the conventionally used Cobb's angle.

We now further develop the appropriate functionality to assist the diagnosis of spinal deformities based on one or more overview images, according to the clinical specifications of the orthopaedic department of the Academic Hospital Groningen, The Netherlands. In a prototype environment, we are developing more advanced measuring functions based on a set of anatomical landmarks of vertebral bodies and pedicles. The localization of these landmarks on a frontal projection image gives access to a set of interesting deformity parameters such as axial rotation, wedge and tilt angles. Similar analyses can be performed using a lateral projection image. Two projections can be 
combined to approximate a three-dimensional vertebrae model.

The access to digital images and the development of a dedicated user interface are meant to replace the time-consuming and unpractical film-based procedures using digitizer tablets. In order to further limit time-consuming and tedious user interaction and to reduce the variability of human observers, some automated processing techniques are explored to assist the landmark extraction. These are the main topics of this paper.
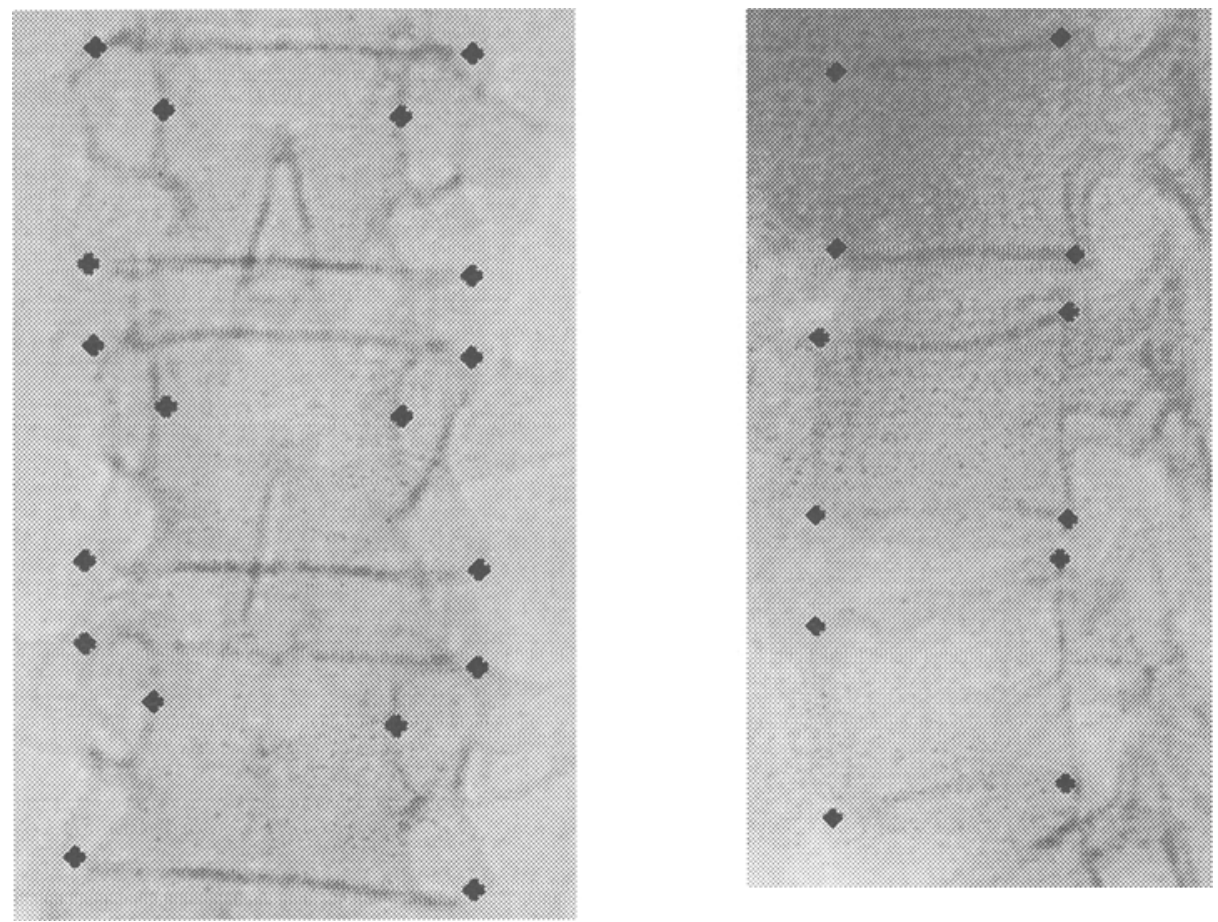

Fig. 2. Vertebral landmarks on a frontal view (4 corner points of the vertebral body and 2 inner edges of the pedicles) and on a lateral view (4 corner points of the vertebral body).

Vertebral body segmentation was also addressed by D'Amico et al. [2] using manual indication of end-plates on gradient direction encoded images. In Kauffman et al. [3] a vertebral body template is first rigidly matched using the generalized Hough transform. These templates are then deformed while optimizing a generalized active contour model energy. We try to develop semi-automated methods that closely cooperate with user interactions and that benefit from the improved image quality of our digital images.

Several authors have described the estimation of 3D models of the human spine, a.o. Stokes [4], Godillon et al. [7] and Dansereau et al. [6] using biplanar orthogonal radiography and André et al. [5] using two vertical stereo radiographs. All of these methods depend on landmark localization on X-ray films using a digitizer tablet or on digitally scanned X-ray films. We have access to digital images directly which simplifies the indication of landmarks considerably thanks to optimal zooming, contrast/brightness correction, edge enhancement, etc. 


\section{Methods}

\subsection{Vertebral landmarks}

Literature uses a blend of different anatomical landmarks on vertebral bodies to capture the geometry of individual vertebrae and of the vertebral column. A frequently used set of landmarks consists of the four corners of the vertebral body in frontal and/or lateral projections and some characteristic points of the pedicles, e.g. the inner edge point (i.e. the most interior point of the pedicle contour) (figure 2). This makes 6 landmarks on a frontal projection and 4 points on a lateral projection.

These landmarks have to be well visible and easily identifiable in the projection images. We therefore avoid the centers of both superior and inferior endplates and the (overall) center of the vertebral body since they cannot be deduced directly from image features. Moreover, these centers can be deduced from the four corner points that are more clearly correlated to image features. In places where the corner points are badly visible, they can sometimes be determined better as the result of the intersection of two line segments: top/bottom plate intersecting with left/right vertebral body sides. If the pedicle contours are entirely delineated, the most interior point can be determined automatically. We do not indicate the spinous process since it is not allways clearly visible and its position is not proportionally related to the degree of scoliosis.

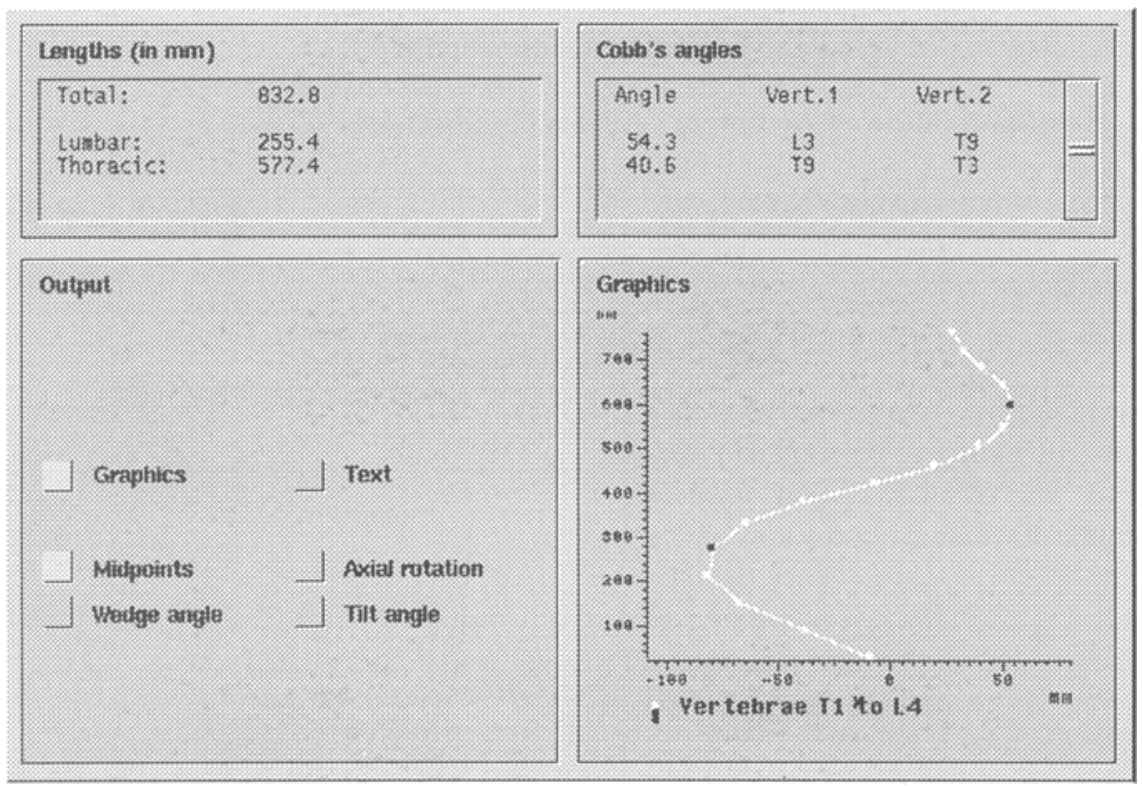

Fig. 3. Analytical parameters of the deformity in a frontal projection, calculated from the set of vertebral landmarks of all vertebrae of the patient in figure 1 . The graph can show different parameters. In this example it represents the coordinates of the midpoints of the vertebral body as projected in the frontal $(\mathrm{x}, \mathrm{z})$ plane (the $\mathrm{z}$ (vertical) and $\mathrm{x}$ (horizontal) coordinates are represented with different scales in the graph). 
We have developed a user interface for indicating these landmarks manually. For each vertebra, the user selects its name (thoracic 1-12, lumbar 1-5) and locates the landmarks in a random order. An algorithm automatically sorts out the meaning of all points using some heuristics on the relative positions of each pair of points.

\subsection{Analytic parameters}

Literature defines all kinds of parameters that assist the orthopaedic surgeon in evaluating the scoliotic deformity. Stokes [8] gives a summary of clear and detailed definitions.

We selected the most significant parameters, calculated them using the vertebral landmarks and summarized them as a set of numbers and charts. An example for the patient on the right of figure 1 is shown in figure 3 .

The output includes both global and local parameters: length of the vertebral axis (total, thoracic and lumbar length), Cobb's angles (with automatic determination of the most inclined vertebrae), coordinates of the midpoints of vertebral bodies (with automatic determination of the apex vertebrae (i.e. the most horizontally displaced vertebrae) ) and three vertebral body angles (axial rotation, wedge and tilt angle). The center of the vertebral body is calculated as the intersection of the line connecting the centers of the superior and inferior endplates with the line connecting the centers of the left and right body sides. Axial rotation is the intrinsic rotation of the vertebral body around its vertical axis and is approximated using Stokes' method [9]. The wedge angle is the angle between the endplates of a vertebral body. The tilt angle is the average angle of both endplates with respect to the horizontal direction.

\subsection{Semi-automatic extraction of vertebral landmarks}

We are currently experimenting with several techniques to speed up and automate the landmark selection process.

Interpolation. After the user has localized landmarks on some key vertebrae, interpolation can be used to estimate the landmark positions on the intermediate vertebrae. Since the user labels the vertebra for which he is entering landmarks, the number of missing vertebrae between each two given vertebrae is known.

This is illustrated in figure 6a where user-indicated points are shown in white. Automatically interpolated landmarks are shown in black. Another example is shown in figure 4 for the entire spine where only 6 out of

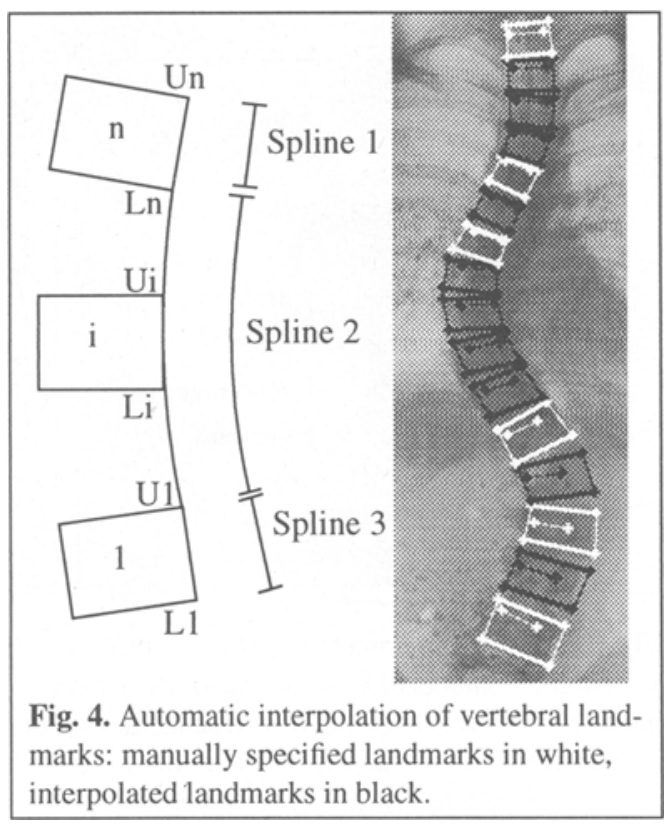


16 vertebrae where localized manually. In this image the corner and pedicle points are connected with a set of lines in order to clarify their meaning.

This interpolation links the corresponding sides of two manually specified vertebrae with a natural cubic spline, formed by three spline segments that have first and second order continuity on connecting points. The positions of the end plates on this spline are defined using the following hypotheses ( $U_{i}$ and $L_{i}$ are curvilinear coordinates):

- the height of successive vertebrae follows a geometrical law, i.e. $U_{i}-L_{i}=r \cdot\left(U_{i-1}-L_{i-1}\right)$ where $r^{n}=\left(U_{n}-L_{n}\right) /\left(U_{1}-L_{1}\right)$;

- the ratio between the height of the vertebrae and the distance between vertebrae is the same.

Vertebral axis. As initialization for further automation, we need to have a global idea of the position and course of the vertebral axis (defined as the line connecting the centers of all vertebral bodies). Since this task is difficult for a computer algorithm but rather easy for a human being, we ask the user to indicate a limited number of points on the spinal axis. These points are interpolated on the fly with a poly-Bezier curve that can be edited interactively (figure 6 b).

Automated global outline detection. Starting from the vertebral axis we extract the global outline of all vertebral bodies as two curves that are more or less parallel to this axis (figure $6 \mathrm{c}$ ). We calculate an oriented edge map (on an appropriate scale) and search for an optimal almost-vertical path using dynamic programming.

The edge detection algorithm chains low-pass recursive filtering of the image with gradient calculation: gradient magnitude and orientation (quantised into 16 directions). An edgeness map is then calculated: only those points for which the projected gradient intensity is a local maximum along the direction of the gradient are given a non-zero edgeness equal to the gradient magnitude.

A path through the edge map is determined which optimizes a cost function that combines constraints on the edge feature strength (edgeness), orientation (gradient orientation with respect to vertebral axis tangent orientation) and path continuity (local path curvature). The path is forced to link strong edge features while remaining approximately parallel to the global vertebral axis.

Automated end plate detection. The edge map also allows to search for a set of lines approximately orthogonal to the global vertebral axis: the vertebral body end-plates. Originally all points on the axis are end-plate candidates. They are qualified according to the quality of an edge path growing out from each of them, orthogonally to the axis.

For this task the gradient image is thresholded with a low threshold in order to capture as many faint edges as possible, resulting in a binary feature image. This image is grey level dilated with a small isotropic structuring element to increase the number of edge point candidates.

For each point on the vertebral axis, one can calculate the cost for linking the current point to the rightmost spine outline and another cost for linking it to the leftmost spine outline. Loosely speaking, each of these costs is the shortest path length linking the current vertebral axis point to one of the spine outlines and going through high gradient 
edge-points. Two paths can thus be determined: one with preferred upward directed gradients, one with downward directed gradients.

Each path is determined in an angular sector as illustrated in figure 5 . Length and slope constraints are imposed to each discretized path representing an endplate candidate. The maximal length is limited to half the approximated width of the vertebrae. The slope angle is forced to remain within $+/-45$ degrees. The cost for having an end-plate going through a vertebral axis point is then defined as the sum of the costs for the left and right paths. This defines the end-plate's "length".

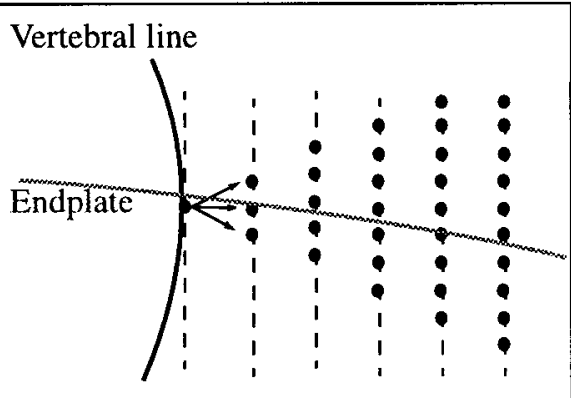

Fig. 5. Endplate detection by path tracking.

This leads to two functions of end plate quality with respect to vertebral axis coordinate: one for end plates with upward gradients, one with downward gradients. Each local minimum of these functions is a candidate end plate position. The user has to indicate the height of the first and the last vertebra and to label them: this allows to determine the number of endplates between both vertebrae and to estimate the approximative height of each vertebra (using a geometric law as described before).

The optimal set of end-plates is then selected with dynamic programming using a cost function that combines the end-plate "length" with the deviation from the expected vertebral body heights. This can be seen as an optimal labeling of all vertebra axis minima to an appropriate vertebra end-plate. End-plates that appear to be missing because they are too far off an expected position, can be filled in by interpolating well detected end-plates. A result of this procedure is illustrated in figure $6 \mathrm{~d}$.

The vertebral body corner points can then be located automatically at the intersection of the endplates with the global vertebral column outlines.

\subsection{D modeling}

A simplified 3D model of the vertebral column can be fitted to two sets of landmarks on frontal and lateral views. The translation reconstructed images closely approximate the orthogonal projections geometry. Therefore these views are only calibrated for differences in scaling and vertical alignment.

A 3D ellipse is fitted to the four landmarks of each vertebral body end-plate while making use of the axial rotation as estimated from the pedicle positions. Top and bottom ellipses are filled with a triangulated surface and the intrinsic orientation is visualized by two tetrahedra that represent the pedicles. This generates a model as illustrated in the three surface rendered views of figure 7 . One of the vertebrae is missing since it was not possible to trace some of the landmarks on the lateral projection image. 


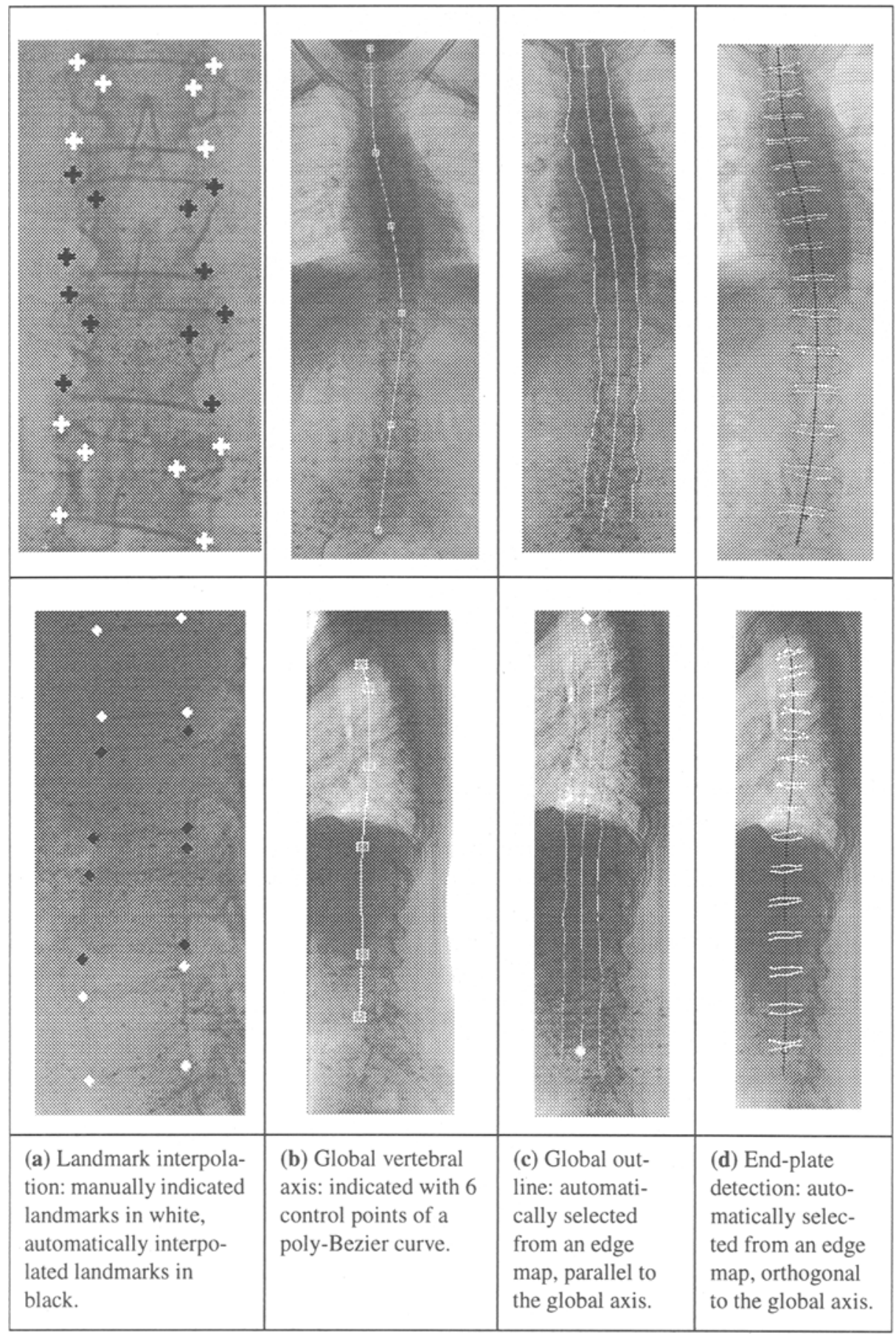

Fig. 6. Steps in the semi-automated localization of vertebra landmarks on frontal (top) and lateral (bottom) views. 


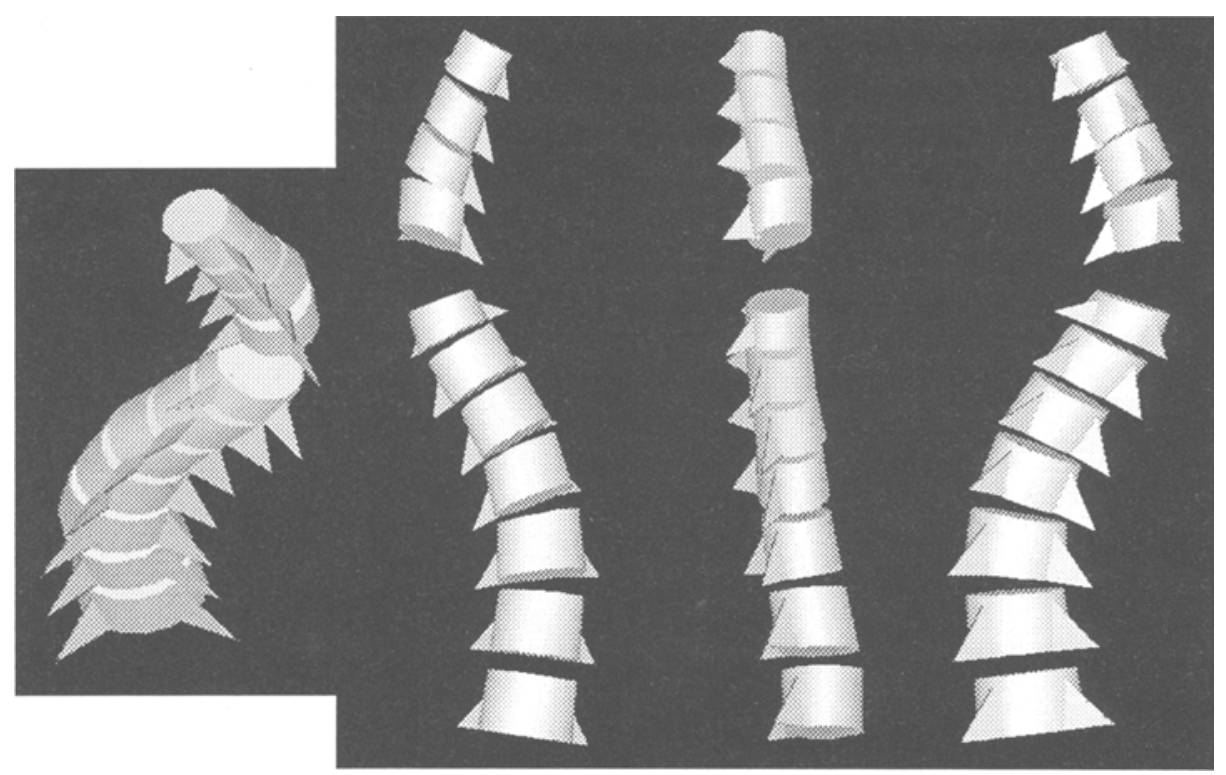

Fig. 7. Triangulated and surface rendered views of the 3D vertebral body model as estimated from the landmarks on a frontal and lateral X-ray projection. One vertebra is missing because it was impossible to trace its landmarks on the lateral view.

\section{Results}

The previous figures illustrated some preliminary results of the analysis and modeling methods that are under development.

The landmark interpolation method shows to facilitate the approximative indication of landmarks in difficult regions. We still need to prove if it really decreases the total interaction time.

The other semi-automated methods appear to perform well but tend to be sensitive to the vertebral axis initialization. Therefore they are currently improved by allowing simple user interactions to correct erroneous results (e.g. small corrections of the global vertebra outline of figure $6 \mathrm{c}$ ). They will then be added to the clinical prototype for further validation.

Image quality of lateral projections is dependent on the degree of the scoliotic deformity: highly laterally curved parts of the vertebral column lead to overprojected vertebrae in the lateral view. Moreover, as the direction of the X-rays is much less parallel to the end plates than in the frontal view, these become poorly visible. The detection of landmarks on lateral projections of high-degree scolioses is thus often problematic.

\section{Discussion}

The overall purpose of this work is to facilitate the analysis of the $3 \mathrm{D}$ deformity of the vertebral column of scoliotic patients. A practical and interactive user interface has been 
developed to indicate vertebral anatomical landmarks easily and to calculate a set of clinically useful parameters. Attempts to locate those landmarks automatically look promising but they remain to be incorporated in an interactive landmark setting procedure and to be extended for pedicle contour extraction.

The reproducibility of manual semi-automated landmark indication is currently investigated by a panel of clinical experts. Additionally, they compare measurements on digital images using the prototype graphical user interface with measurements from digitized landmarks on conventional X-ray films. These studies should establish the accuracy and reliability of the proposed methods.

Due to the inherent problems of lateral acquisitions we are looking for other imaging protocols that are dedicated to 3D modeling and that could result in more clearly identifiable landmarks.

\section{Acknowledgments}

The authors thank N. Pivet and P. Lacour for their wonderful trainee-work. We acknowledge the inspiration of $\mathrm{A}$. van Eeuwijk and S. Lobregt during the start up phase of this research project.

\section{References}

1. A. H. W. van Eeuwijk, S. Lobregt and F.A. Gerritsen: A novel method for digital Xray imaging of the complete spine", Proceedings of CVRMed-MRCAS'97, Lecture Notes in Computer Science vol. 1205, pp. 521-530, 1997.

2. M. D'Amico, S. Dell'era, P. Roncoletta: New procedure for computation and automatic classification of spinal clinical parameters by image processing of digitised radiography, Three dimensional analysis of spinal deformities, M. D'Amico et al. (Eds.), pp. 39-43, 1995.

3. C. Kauffman, J.A. de Guise: Digital radiography segmentation of scoliotic vertebral body using deformable models, SPIE, vol. 3034, pp.243-251, 1997.

4. I.A.F. Stokes: Biplanar radiography for measurement of spinal shape and motion, Automedica, Vol. 5, pp. 37-49, 1985.

5. B. André, J. Dansereau, H. Labelle: Optimized vertical stereo base radiographic setup for the clinical three-dimensional reconstruction of the human spine, J. Biomechanics, vol. 27:8, pp. 1023-1035, 1994.

6. J. Dansereau, A. Chabot, N.T. Huynh, H. Labelle, J. de Guise: 3-D reconstruction of vertebral endplate wedging, Three dimensional analysis of spinal deformities, $\mathbf{M}$. D'Amico et al. (Eds.), pp. 69-73, 1995.

7. A.P. Godillon, F.X. Lepoutre, D. Chopin: Evaluation of a 3D geometrical spinal model, Three dimensional analysis of spinal deformities, M. D'Amico et al. (Eds.), pp. 191-195, 1995.

8. I. A. F. Stokes: Three-dimensional terminology of spinal deformity, SPINE, vol. 19:2, pp. 236-248, 1994.

9. I.A.F. Stokes, L.C. Bigalow and M.S. Moreland: Measurement of axial rotation of vertebrae in scoliosis, Spine, Vol. 11:3, pp. 213-218, 1986. And a correction in a "letter to the editor" in Spine, Vol. 16:5, 1991. 